\title{
Quantitative Determination of Lactones in Piper methysticum (Kava-Kava) by Supercritical Fluid Chromatography*
}

Authors

Adele Murauer, Markus Ganzera

Affiliation

Institute of Pharmacy, Pharmacognosy, University of Innsbruck, Innsbruck, Austria

Key words

supercritical fluid chromatography, Piper methysticum, Piperaceae, kavalactones

received November 29, 2016

revised December 20, 2016

accepted January 9, 2017

Bibliography

DOI http://dx.doi.org/10.1055/s-0043-100632

Published online January 17, 2017 | Planta Med 2017; 83:

1053-1057 @ Georg Thieme Verlag KG Stuttgart · New York | ISSN 0032-0943

Correspondence

Assoc. Prof. Dr. Markus Ganzera

Institute of Pharmacy, Pharmacognosy, University of

Innsbruck

Innrain 80-82, 6020 Innsbruck, Austria

Phone: + 4351250758406 , Fax: + 4351250758499

markus.ganzera@uibk.ac.at
$\Theta$ Supporting information available online at http://www.thieme-connect.de/products

\section{ABSTRACT}

A fast and validated supercritical fluid chromatography method for the quantitative determination of major lactones in Piper methysticum, a plant used against nervous anxiety, stress, and restlessness, was developed. The baseline separation of dihydrokavain, demethoxyyangonin, kavain, yangonin, dihydromethysticin, and methysticin was possible in less than 4 min on an Aquity UPC ${ }^{2}$ BEH $1.7 \mu$ m column, in combination with a mobile phase comprising $\mathrm{CO}_{2}$ and methanol with diethylamine. The column temperature had a great impact on the results because only at $70^{\circ} \mathrm{C}$ could kavain and yangonin be fully resolved. With correlation coefficients above 0.998 , recovery rates between 95.9 and $104.1 \%$ as well as limit of detection values below $1.5 \mathrm{ng}$ on-column, the procedure fulfilled all validation requirements and was well suited for the quantitative analysis of commercial products containing $P$. methysticum root powder and/or extract. All of them contained the target analytes, however, the absolute content of lactones was quite variable. Accordingly, depending on the product, the total daily intake of lactones varied from 56 to $312 \mathrm{mg}$. Concerning speed, selectivity, and environmental friendly operation, this supercritical fluid chromatography approach surpasses all previously reported ones.

\section{Introduction}

On many Pacific islands like Samoa, Hawaii, or Fiji, the rhizomes and roots of Piper methysticum Forst. (Piperaceae) are traditionally used to prepare a ceremonial and relaxing beverage known as kava or awa [1]. Also in the Western world, kava products enjoyed great popularity for many years as an herbal remedy to treat mild to moderate forms of situational anxiety, an activity that is associated with several lactones present in the plant [2]. The ban of respective products in Europe 2002 was surprising, because their assumed hepatotoxicity could not be confirmed convincingly. Several possible explanations for reported liver damage after kava consumption were postulated, ranging from human genetic variability and the use of inappropriate solvents for extraction to plant

* Dedicated to Professor Dr. Max Wichtl in recognition of his outstanding contribution to pharmacognosy research. adulteration [3]. As a consequence, in February 2015, the German upper administrative court confirmed a previous decision that a ban of kava is not justified because of indecisive data on its toxicity or efficacy. The latter is still under discussion as respective information is controversial to a large extent [4-6]. However, recent studies point to other interesting bioactivities of kavalactones as well, such as anticancer [7,8] or antihyperglycemic [9] properties.

Because of the aforementioned issues, the analysis of kavalactones has been an important topic in analytical sciences for a long time. More than 15 years ago we developed and compared different techniques (normal- and reversed-phase HPLC, GC) for their determination in plant material [10]; Bilia et al. [11] prepared an excellent review on the same topic in 2004. More recent studies report on the comparison of different kava varieties by HPTLC [12], analyzed respective food supplements by LC-MS [13], or used NIR for quantification and authentication [14]. Except of one paper focusing on the extraction of $P$. methysticum by supercritical $\mathrm{CO}_{2}$ [15], the use of supercritical fluid chromatography (SFC) for the separation of kavalactones has only been reported once [16]. The 
study was already conducted 1999 so that the described instrumental setup and the achieved resolutions do not reflect the current possibilities of this emerging separation technique anymore. Furthermore, as a semipreparative application is described, method validation and quantitative results are missing. These facts, together with an impressive potential and versatility for natural products analysis [17-19], motivated us to evaluate the separation of kavalactones in $P$. methysticum by SFC again.

\section{Results and Discussion}

Environmental and theoretical advantages of SFC are a convincing argument [17], however, practically relevant applications will promote its use even further. Especially those related to natural products analysis are a good indicator of separation efficiency, because the investigated matrices are usually complex and the determined analytes often share a high degree of structural similarity. In the last few years, the number of SFC reports for natural products analysis has increased steadily, yet the majority of relevant plant species has never been investigated, or respective publications are comparatively old and do not reflect the recent improvements in instrumentation and stationary phases. The SFC analysis of kavalactones is an example of the latter.

The optimized SFC separation of six kavalactones [dihydrokavain (1), demethoxyyangonin (2), kavain (3), yangonin (4), dihydromethysticin (5) and methysticin (6); for respective structures see > Fig. 1] is shown in > Fig. 2 A. However, their baseline resolution in less than 4 min was only possible after carefully investigating the impact of diverse separation parameters. Most important in this respect was the choice of stationary phase, mobile phase composition, and separation temperature. While searching for the optimum column, eight different Aquity SFC columns from Waters with identical dimensions $(3.0 \times 100 \mathrm{~mm})$ were tested, more precisely, they were BEH 1.7 $\mu \mathrm{m}$, BEH 2-EP $1.7 \mu \mathrm{m}$, CSH Fluoro Phenyl $1.7 \mu \mathrm{m}$, HSS C18 SB $1.8 \mu \mathrm{m}$, and four Torus phases (2-PIC 1.7 $\mu \mathrm{m}$, Diol 1.7 $\mu \mathrm{m}$, DEA $1.7 \mu \mathrm{m}$ and 1-AA 1.7 $\mu \mathrm{m}$ ). Only on unbonded ethylen bridged hybrid material $(\mathrm{BEH})$, the first stationary phase mentioned, could all six standard compounds be resolved satisfactorily. Others like 2-ethylpyridine (2-EP) modified $\mathrm{BEH}$, which has a less polar surface, resulted in partial peak overlapping ( $\vee$ Fig. 2 B). This indicated that a stronger interaction with polar functional groups of the analytes was favorable to achieve better results. However, only using supercritical $\mathrm{CO}_{2}$ as the mobile phase was not possible due to its insufficient solvent strength. It is therefore common practice to use a solvent gradient by increasingly adding organic modifiers such as isopropanol, acetonitrile, or methanol. The latter is the most common one, and also for the current application it showed the best results. To further improve peak shape and resolution, diethylamine was added to methanol ( $\vee$ Fig. $2 \mathrm{C}$ ), with a concentration of $0.6 \%$ being the optimum. Using an acid (acetic or phosphoric acid) or buffer salt (ammonium acetate) instead resulted in no improvement or even deteriorated the results. The third parameter with significant influence on the separation of kavalactones was temperature. The finally selected $70^{\circ} \mathrm{C}$ was rather high for SFC and already $10^{\circ} \mathrm{C}$ above the maximum recommended by the manufacturer. How-

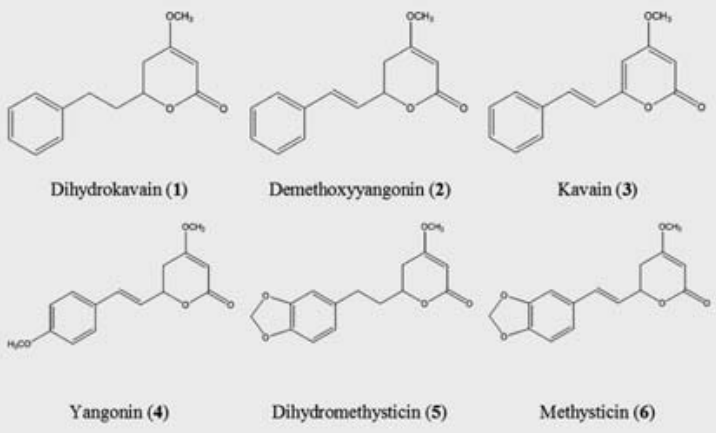

- Fig. 1 Chemical structure of the six kavalactones analyzed in this study.
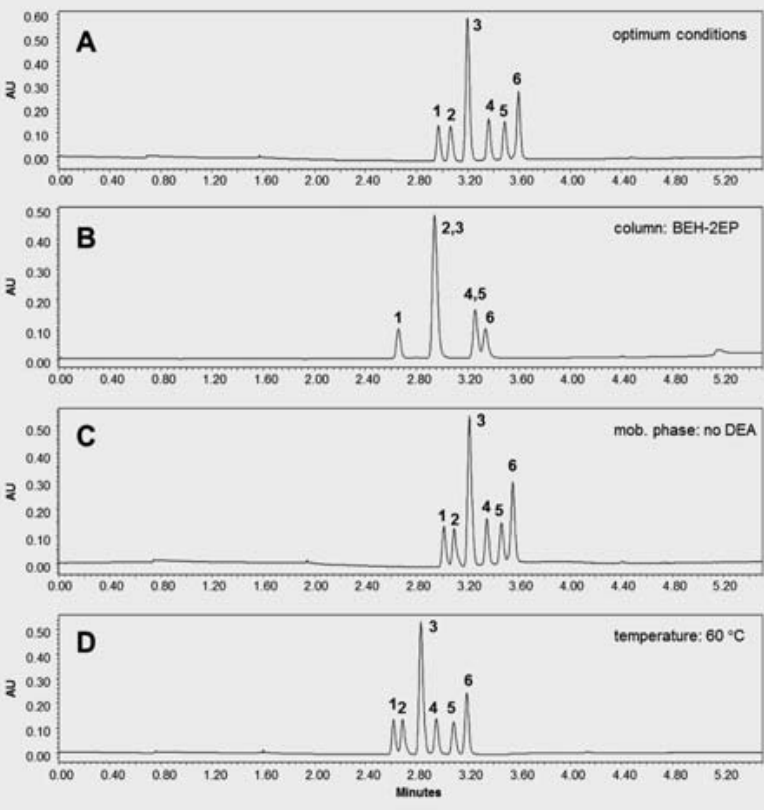

- Fig. 2 Optimized SFC separation of standard compounds (A), and the impact of a different stationary phase (B), a mobile phase without diethylamine as an additive (C), and a column temperature of $60^{\circ} \mathrm{C}$ (D) on the results; all other settings were optimal. The assignment of peaks is according to $>$ Fig. 1 .

ever, compared to $60^{\circ} \mathrm{C}$ the separation was significantly better ( $\vee$ Fig. 2D), and we observed no decline in column performance and separation efficiency even after approx. 500 injections. If analyses are to be performed at lower column temperatures, it should be noted that only from $50{ }^{\circ} \mathrm{C}$ upwards compounds 3 and 4 gradually start to separate, below this point they completely coelute. In respect to ABPR (automated backpressure regulator) and flow rate, the finally selected values of $1885 \mathrm{psi}$ and $1 \mathrm{~mL} /$ min permitted fast separations and did not exceed the pressure limit of the instrument (5800 psi or 400 bar). Both settings only had a limited influence on the overall separation, except that a 
- Table 1 Summary of the validation results.

\begin{tabular}{|c|c|c|c|c|c|c|}
\hline \multirow[t]{2}{*}{ Parameter } & \multicolumn{6}{|l|}{ Compound } \\
\hline & 1 & 2 & 3 & 4 & 5 & 6 \\
\hline Regr. equation & $\begin{array}{l}y=1452.5 x+ \\
798.8\end{array}$ & $\begin{array}{l}y=1596.5 x- \\
1550.3\end{array}$ & $\begin{array}{l}y=5705.7 x- \\
467.2\end{array}$ & $\begin{array}{l}y=1633.0 x+ \\
998.1\end{array}$ & $\begin{array}{l}y=2239.5 x- \\
1934.5\end{array}$ & $\begin{array}{l}y=2299.7 x+ \\
1409.3\end{array}$ \\
\hline $\mathbf{R}^{2}$ & 0.9988 & 0.9985 & 0.9987 & 0.9985 & 0.9994 & 0.9994 \\
\hline Linearity $^{\mathrm{a}}$ & $730-5.7$ & $680-5.3$ & $730-5.7$ & $730-5.7$ & $780-6.1$ & $800-6.2$ \\
\hline LOD $^{a}$ & 0.6 & 1.1 & 0.6 & 1.1 & 1.4 & 1.4 \\
\hline $\operatorname{LOQ}^{\mathrm{a}}$ & 1.9 & 3.4 & 1.9 & 3.2 & 4.1 & 4.3 \\
\hline \multicolumn{7}{|l|}{ Precision } \\
\hline - intraday ${ }^{b}$ & 0.9 & 1.6 & 1.3 & 0.9 & 1.0 & 0.6 \\
\hline - interdayc & 0.4 & 3.6 & 1.4 & 1.8 & 0.8 & 1.0 \\
\hline \multicolumn{7}{|l|}{ Accuracy $^{d}$} \\
\hline - high spike & 95.9 & 101.0 & 98.0 & 104.1 & 96.1 & 99.0 \\
\hline - medium spike & 99.9 & 98.0 & 99.2 & 96.9 & 97.4 & 100.6 \\
\hline - low spike & 98.6 & 97.2 & 99.2 & 101.0 & 97.6 & 100.8 \\
\hline
\end{tabular}

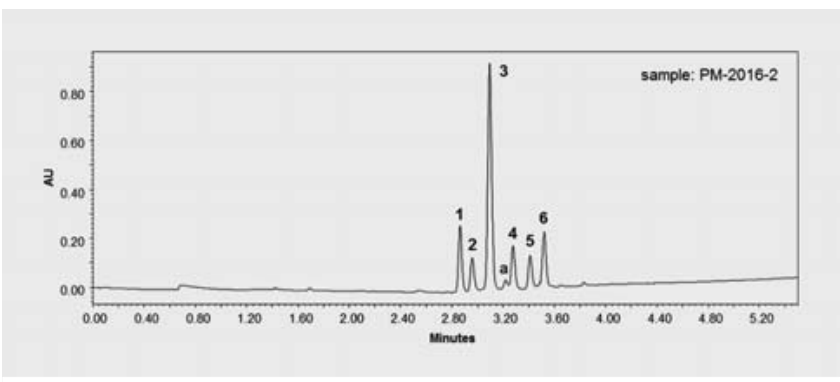

- Fig. 3 Analysis of a commercial kava product (sample PM-20162) by SFC [column: Aquity UPC ${ }^{2}$ BEH $1.7 \mu \mathrm{m}, 3.0 \times 100 \mathrm{~mm}$; mobile phase: $\mathrm{CO}_{2}(\mathrm{~A}), 0.6 \%$ diethylamine in methanol (B); gradient: $100 \mathrm{~A} /$ $O B$ in 3 min to $86 \mathrm{~A} / 14 \mathrm{~B}$, then in 2 min to $80 \mathrm{~A} / 20 \mathrm{~B}$ and kept at this composition for $0.5 \mathrm{~min}$; sample volume: $1 \mu \mathrm{L}$; flow rate: $1 \mathrm{~mL} / \mathrm{min}$; column temperature: $70^{\circ} \mathrm{C}$; ABPR: 1885 psi; detection: $240 \mathrm{~nm}$ ]. The assignment of compounds is according to $>$ Fig. 1.

slightly higher flow rate (e.g., $1.2 \mathrm{~mL} / \mathrm{min}$ ) already resulted in a reduced resolution between 5 and 6 .

The developed method was validated according to ICH guidelines [20] in order to confirm that it is suitable for its intended use, which is the quantitative determination of major kavalactones in commercial products. Results of all respective experiments are combined in $>$ Table 1 . Calibration experiments showed that within a concentration range of approx. $6-750 \mu \mathrm{g} / \mathrm{mL}$, the detector response was linear $\left(R^{2} \geq 0.9985\right)$ for all six standards. Limit of detection (LOD) and limit of quantitation (LOQ) were found to be less or equal to 1.4 and $5.3 \mu \mathrm{g} / \mathrm{mL}$, which corresponds to 1.4 and $5.3 \mathrm{ng}$ injected on-column. Selectivity was confirmed by two factors. First, there was no visible coelution (shoulder) in the signals of interest, and second, DAD data were highly consistent as eval- uated with the peak purity option of the operating software. A final confirmation of selectivity by MS was not possible as the respective coupling is currently not available in our lab. Intermediate precision for the repeated determination (extraction and analysis) of lactones in sample PM-2016-1 revealed maximum deviations of $1.6 \%$ (compound 2, intraday) and 3.6\% (compound 2, interday). Recovery rates indicating the methods accuracy were satisfactory as well, as they ranged from 95.9\% (compound 1, high spike) to $104.1 \%$ (compound 4 , high spike).

Five dietary supplements containing $P$. methysticum root powder and/or extract were analyzed by SFC, and a typical chromatogram is shown in - Fig. 3. In the course of these investigations, the applied extraction procedure was evaluated for efficiency. One sample (PM-2016-2) was extracted as proposed (see Materials and Methods - Sample preparation). Then the remaining solid material was extracted once more, and the obtained solution was assayed for any remaining lactones. As no quantifiable amounts of 1 to 6 were found, the extraction protocol was considered exhaustive and thus suitable for performing quantitative studies.

As can be seen from the compiled results shown in $\mathbf{r}$ Table $\mathbf{2}$, all six reference compounds could be determined in each of the products; repeatability of the assay was satisfying with a maximum relative standard deviation of $2.62 \%(n=3)$. The compounds were assigned in the samples by matching retention times and UV spectra in comparison to standards (Supporting Information); some of the extracts contained an additional signal (a) between 3 and 4, whose UV spectra was similar to yangonin. It can be speculated that this compound might be tetrahydroxyyangonin [11], however, without a respective standard its identity remains unknown. In the solid products, the major lactones were dihydrokavain (1, 2.06-7.28\%), yangonin (4, 1.98-4.64\%), and kavain (3, $1.87-7.09 \%$ ); dihydromethysticin $(5,0.68-1.67 \%)$ was always present in the lowest amounts. The two liquid formulations, PM- 
- Table 2 Quantitative determination of kavalactones in different dietary supplements by SFC, including the total daily intake (TDI) based on the maximum dose recommended by the manufacturers; relative standard deviation in parentheses $(n=3)$.

\begin{tabular}{|c|c|c|c|c|c|}
\hline Compound & PM-2016-1 & PM-2016-2 & PM-2016-3a & PM-2016-4 & PM-2016-5 \\
\hline 1 & $2.06(0.44)$ & $7.28(0.88)$ & $2.83(1.37)$ & $10.14(0.63)$ & $12.18(0.46)$ \\
\hline 2 & $1.01(0.40)$ & $3.50(1.64)$ & $1.39(0.55)$ & $5.23(0.39)$ & $7.08(0.32)$ \\
\hline 3 & $1.87(0.38)$ & $7.09(1.22)$ & $2.62(1.08)$ & $10.09(0.64)$ & $11.68(0.36)$ \\
\hline 4 & $1.98(0.38)$ & $4.64(2.62)$ & $2.03(1.15)$ & $5.80(0.22)$ & $24.10(0.85)$ \\
\hline 5 & $0.68(0.28)$ & $1.67(1.31)$ & $0.80(0.85)$ & $2.63(0.22)$ & $4.81(0.61)$ \\
\hline 6 & $1.25(0.31)$ & $3.29(1.06)$ & $1.33(0.31)$ & $5.19(0.40)$ & $6.69(0.18)$ \\
\hline$T D K^{k}$ & 56.66 & 312.05 & 193.74 & 195.40 & 242.98 \\
\hline
\end{tabular}

a Solid product, values reflect percent (mg lactone/100 mg product). ${ }^{\mathrm{b}}$ Liquid product, values reflect $\mathrm{mg}$ lactone/mL product. ${ }^{\mathrm{C}}$ Total kavalactones in $\mathrm{mg} /$ day.

2016-4 and PM-2016-5, followed the same pattern, except that in the latter the content of yangonin was extremely high at $24.10 \mathrm{mg} / \mathrm{mL}$. Demethoxyyangonin (2) and methysticin (5) were found in concentrations ranging from 5.23 to 7.08 and 5.19 to $6.69 \mathrm{mg} / \mathrm{mL}$, respectively. In order to evaluate the products from a consumer's point of view, the total daily intake, based on the maximum dose recommended, was calculated. With product PM-2016-1, 57 mg kavalactones are ingested, whereas PM-20162 supplies more than $312 \mathrm{mg}$ per day. That this more than 5 -fold difference might result in divergent (side) effects is therefore not unlikely.

When comparing the here described method with others previously reported for the analysis of kavalactones, significant advantages are obvious. Only one related SFC assay was published nearly two decades ago, and a direct comparison seems unfair considering today's technical possibilities. Anyway, not only was the analysis time, depending on the utilized columns, at least three times longer then and no baseline separation of the major lactones was achieved, but also validation and quantification were missing [16]. Also, by capillary electrophoresis (13 min), gas chromatography (18 min), or conventional RP-HPLC (27 min), the separation of kavalactones requires significantly more time [11]. In a recent LC-MS study [13], a protocol for simultaneously characterizing 79 medicinal plants was proposed. P. methysticum was one of them, however, this study focused on the identification of kavain only and quantitative results were not presented. In this respect, our SFC procedure permits an extremely fast (baseline) separation of all major lactones in crude plant material and commercial products. All validation criteria are fulfilled in the most satisfying way, and quantitative results are reproducible and in agreement with published data. For example, in our initial study, we found quite a variable content of total lactones in the drug ranging from approx. 2 to $15 \%$, with kavain and dihydrokavain being the dominant compounds [10]. In the current work, these two were also major compounds, and product PM-2016-1, which only consisted of pure $P$. methysticum root powder, contained $8.85 \%$ lactones. Accordingly, for the analysis of kavalactones, SFC should not only be considered an equivalent alternative but probably the method of choice, with its surpassed separation speed and selectivity as well its environmental friendly nature.

\section{Materials and Methods}

\section{Standards and reagents}

Six kavalactones (compounds 1-6), all with a purity $\geq 98.4 \%$, were available as standards from Phytolab. The analyzed commercial samples PM-2016-1 to PM-2016-5 were bought in 2016 in diverse health food stores in Annapolis, MD, USA. Compressed carbon dioxide for SFC analysis had a purity of $\geq 99.995 \%$ (4.5 grade) and came from Messer. All solvents and reagents (methanol, acetonitrile, isopropanol, diethylamine) utilized in this study were of analytical grade and purchased from Merck.

\section{Sample preparation}

The commercial samples analyzed either contained a solid (PM2016-1: ground P. methysticum root powder; PM-2016-2 and 3: a mixture of kava root powder and supercritical root extract) or were liquids containing an ethanolic root extract (PM-2016-4 and 5). The solid samples were prepared as follows: one capsule was opened and its content weighed and extracted three times with $10 \mathrm{~mL}$ methanol by sonication for $20 \mathrm{~min}$ each. After centrifugation with $700 \times g$ for 6 min, the supernatants were collected in a $100-\mathrm{mL}$ volumetric flask and the latter filled to volume with methanol. For liquid products, $1 \mathrm{~mL}$ was directly diluted to $100.00 \mathrm{~mL}$ with methanol. All sample solutions were membrane filtered before injection, using ProFill cellulose HPLC syringe filters with a $0.45 \mu \mathrm{m}$ pore size obtained from Bruckner, and analyzed in triplicate. Voucher specimens of all products (PM-2016-1: LOT 150800114; PM-2016-2: LOT 201523050; PM-2016-3: LOT A100601003; PM-2016-4: LOT 3521800; PM-2016-5: LOT 410461) are deposited at the Institute of Pharmacy, Pharmacognosy, University of Innsbruck.

\section{Analytical method}

For all experiments, an Aquity UPC ${ }^{2}$ SFC instrument from Waters, equipped with a convergence, column, sample, binary solvent 
manager and PDA detector was used; the operating software was Empower 3. Optimum separation of the kavalactones was achieved on an Aquity UPC ${ }^{2}$ BEH $1.7 \mu \mathrm{m}$ column $(3.0 \times 100 \mathrm{~mm}$; Waters), protected by a $0.2-\mu \mathrm{m}$ guard filter (critical clean; Waters). The mobile phase comprised $\mathrm{CO}_{2}(\mathrm{~A})$ and $0.6 \%$ diethylamine in methanol (B). Gradient elution started with $100 \mathrm{~A} / 0 \mathrm{~B}$, followed by $86 \mathrm{~A} / 14 \mathrm{~B}$ in $3 \mathrm{~min}$, and $80 \mathrm{~A} / 20 \mathrm{~B}$ in another $2 \mathrm{~min}$; this composition was kept for half a minute (total run time $5.5 \mathrm{~min}$ ). The column was then re-equilibrated for 2 min under initial separation conditions. The injected sample volume was $1 \mu \mathrm{L}$, and flow rate, column temperature, and ABPR pressure were set to $1 \mathrm{~mL} / \mathrm{min}, 70^{\circ} \mathrm{C}$, and 1885 psi (= 130 bar), respectively. The compounds of interest were detected at $240 \mathrm{~nm}$. Temperature of the sample manager was set to $10{ }^{\circ} \mathrm{C}$, and a mixture of methanol/2-propanol $(1: 1)$ and methanol served as a weak and strong wash, respectively.

\section{Method validation}

Validation experiments were carried out to study the performance characteristics of the developed SFC procedure. In order to establish calibration curves and to determine LOD and LOQ values, a stock solution of all compounds in methanol (concentration $750 \mathrm{mg} / \mathrm{mL}$ each) was prepared. This solution was serially diluted with the same solvent in the ratio of $1: 1$, and LOD (signal-to-noise ratio 3) and LOQ (S/N ratio 10) were visually evaluated. To confirm selectivity, DAD data of each relevant signal and the peak purity option in the operating software were utilized. Intermediate precision was assured by analyzing five individually prepared solutions of sample PM-2016-1 on day 1. The same procedure was repeated on two more days, and the variation within one day (intraday precision) and within three days (interday precision) was calculated based on the peak area. Accuracy was investigated by spiking sample PM-2016-1 with different concentrations of each standard compound (high, medium, and low spike). The spiked samples were then extracted and analyzed with the optimized SFC method. The recovery rate was determined by comparing the theoretically present with the practically found amount for each of the standards. All standard and sample solutions prepared during method validation were analyzed in triplicate.

\section{Supporting information}

A comparison of the UV spectra of six kavalactone standards and respective signals in a sample solution is available as Supporting Information.

\section{Conflict of Interest}

The authors declare no conflict of interest.

\section{References}

[1] Singh YN. Kava: an overview. J Ethnopharmacol 1992; 37: 13-45

[2] Ma Y, Sachdeva KL, Lui J, Ford M, Yang D, Khan IA, Chichester CO, Yan B. Desmethoxyyangonin and dihydromethysticin are two major kavalactones with marked activity on the induction of CYP3A23. Drug Metab Dispos 2004; 32: 1317-1324
[3] Kuchta K, Schmidt M, Nahrstedt A. German kava ban lifted by court: the alleged hepatotoxicity of kava (Piper methysticum) as a case of ill-defined herbal drug identity, lacking quality control, and misguided regulatory politics. Planta Med 2015; 81: 1647-1653

[4] Sarris J, Scholey A, Schweitzer I, Bousman C, LaPorte E, Ng C, Murray G, Stough $C$. The acute effects of kava and oxacepam on anxiety, mood, neurocognition and genetic correlates: a randomized, placebo-controlled, double-blind study. Hum Psychopharmacol 2012; 27: 262-269

[5] Garrett KM, Basmadjian G, Khan IA, Schaneberg BT, Searle TW. Extracts of kava (Piper methysticum) induce acute anxiolytic-like behavioral changes in mice. Psychopharmacology (Berl) 2003; 170: 33-41

[6] Gastpar M, Klimm HD. Treatment of anxiety, tension and restlessness states with kava special extract WS 1490 in general practice; a randomized placebo-controlled double-blind multicenter trial. Phytomedicine 2003; 10: 631-639

[7] Tang SN, Zhang J, Jiang P, Datta P, Leitzman P, O'Sullivan MG, Jiang C, Xing $C$, Lue J. Gene expression signatures associated with suppression of TRAMP prostate carcinogenesis by a kavalactone-rich kava fraction. Mol Carcinog 2016; 55: 2291-2303

[8] Narayanapillai SC, Balbo S, Leitzmann P, Grill AE, Upadhyaya P, Shaik AA, Zhou B, O'Sullivan MG, Peterson LA, Lu J, Hecht SS, Xing C. Dihydromethysticin from kava blocks tobacco carcinogen 4-(methylnitrosamino)-1-(3-pyridyl)-1-butanone-induced lung tumorigenesis and differentially reduces DNA damage in A/J mice. Carcinogenesis 2014; 35: 23652372

[9] Abdel Jaleel GA, Saleh DO, El-Awdan SA. Antihyperglycaemic effect of kava-kava (Piper methysticum) in streptozotocin-induced diabetic rats. Pharm Lett 2013; 5: 104-110

[10] Ganzera M, Khan IA. Analytical techniques for the determination of lactones in Piper methysticum Forst. Chromatographia 1999; 50: 649-653

[11] Bilia AR, Scalise L, Bergonzi MC, Vincieri FF. Analysis of kavalactones from Piper methysticum (kava-kava). J Chromatogr B 2004; 812: 203-214

[12] Lebot V, Legendre L. Comparison of kava (Piper methysticum Forst.) varieties by UV absorbance of acetonic extracts and high-performance thinlayer chromatography. J Food Compos Anal 2016; 48: 25-33

[13] Mathon C, Duret M, Kohler M, Edder P, Bieri S, Christen P. Multi-targeted screening of botanicals in food supplements by liquid chromatography with tandem mass spectrometry. Food Chem 2013; 138: 709-717

[14] Lasme P, Davrieux F, Montet D, Lebot V. Quantification of kavalactones and determination of kava (Piper methysticum) chemotypes using nearinfrared reflectance spectroscopy for quality control in Vanuatu. J Agric Food Chem 2008; 56: 4976-4981

[15] Lopez-Avila V, Benedicto ]. Supercritical fluid extraction of kava lactones from Piper methysticum (kava) herb. J High Resolut Chrom 1997; 20: 555-559

[16] Ashraf-Khorassani M, Taylor LT, Martin M. Supercritical fluid extraction of kava lactones from kava root and their separation via supercritical fluid chromatography. Chromatographia 1999; 50: 287-292

[17] Hartmann A, Ganzera M. Supercritical fluid chromatography - theoretical background and applications on natural products. Planta Med 2015; 81: 1570-1581

[18] Pfeifer I, Murauer A, Ganzera M. Determination of coumarins in the roots of Angelica dahurica by supercritical fluid chromatography. J Pharmaceut Biomed 2016; 129: 246-251

[19] Aichner D, Ganzera M. Analysis anthraquinones in rhubarb (Rheum palmatum and Rheum officinale) by supercritical fluid chromatography. Talanta 2015; 144: 1239-1244

[20] International Conference on Harmonization of technical Requirements for the Registration of Pharmaceuticals for Human Use - Validation of analytical Procedures Q2(R1). Available at http://www.ich.org. Accessed December 19, 2016 